\title{
Studies on swelling of wood with water and ionic liquids
}

\author{
P. Höhne ${ }^{1} \cdot$ K. Tauer ${ }^{1}$
}

Received: 28 April 2015/Published online: 13 November 2015

(C) The Author(s) 2015. This article is published with open access at Springerlink.com

\begin{abstract}
A recently developed simple method to qualitatively study the swelling behavior of polymeric gels is applied to swelling studies of wood with water and ionic liquids. Results are presented showing the differences between and the peculiarities of both kinds of swelling agents. In proof-of-principle experiments, it is shown that swelling of wood with ionic liquids allows the construction of longlasting connections and joints.
\end{abstract}

\section{Introduction}

Wood is a unique substance. Due to its properties, renewability, and abundance around the world, it is one of the oldest natural resources and in use already by the early mankind as engineering material (Chowdhury 1970). It is like all living matter predestined for functioning under wet or humid conditions but also prone to biological degradation (Rowell 2005). As long as wood is used as construction material, its dimensional changes in dependence on swelling and deswelling with the degree of humidity are known and challenge human ingenuity. Wood swells anisotropically-largest size change in the direction of the annual growth rings (tangentially), less crosswise to the rings, and little in direction of the stem. In addition, different wood types swell differently (Rowell et al. 2005). Several preservation strategies are known to protect wood against biological degradation (Schultz et al. 2007) and to control or reduce the water-uptake of wood (Evans

Electronic supplementary material The online version of this article (doi:10.1007/s00226-015-0779-8) contains supplementary material, which is available to authorized users.

P. Höhne

patrick.hoehne@mpikg.mpg.de

1 Max Planck Institute of Colloids and Interfaces, 14424 Potsdam, Germany 
2003). Among them are impregnation, coating, and modification of wood with hydrophobic compounds (Rowell and Banks 1985). During the centuries, the wood protection strategies have changed in dependence on the developments in chemistry, societal needs, environmental concern, and the improving knowledge of wood structure and its functions (Freeman et al. 2003).

The degree of swelling with liquids under ambient conditions is subject to all external changes with respect to humidity and temperature. Cycles of temperature and humidity modify the swelling status of wood and induce mechanical stress on wooden constructions. This can lead on the one hand to issues regarding the overall reliability and usability of wooden constructions; on the other hand, in order to minimize problematic effects, special measures are necessary.

Since more than a decade, it is known that ionic liquids (ILs) are non-derivatizing solvents for cellulose, particularly at elevated temperatures (Swatloski et al. 2002). Subsequently, ILs have been studied for biorefinery of cellulose-containing biomass and as solvents for modification of cellulose (Gericke et al. 2012). Cellulosic materials are the main components in dry wood (up to $70 \mathrm{wt} \%$ ), and hence studying the application of ionic liquids to wood is straightforward. Benefits are expected with respect to preservation, antistatic finishing, and fire-proof properties of wooden products (Han et al. 2009).

Dissolution of polymers requires, as first step, always swelling of the contact region with the solvent (Narasimhan and Peppas 1997), and consequently the application of ILs for the modification of wood involves inevitably the consideration of swelling. The issue is extremely complex because wood is an anisotropic composite material and the various components (mainly lignin and holocellulose) interact differently with a given IL. In addition, due to the heterogeneity also of wood pieces from the same source, experiments, particularly with smaller wood pieces, require always several repeats to obtain significant results.

Of course, the observable changes in the interaction between wood and IL may vary for different wood species and ILs. In a recent study, it was shown that 1-ethylpyridinium bromide reacts preferentially with lignin and the crystalline cellulose is not affected (Kanbayashi and Miyafuji 2015). The treatment of wood with ILs causes changes in the morphology of the anisotropic structure of wood tissues (Miyafuji and Suzuki 2012; Kanbayashi and Miyafuji 2013, 2014).

Swelling of wood with IL was also used to modify the composition of wood and to introduce enzymes or catalysts for enhanced reactivity during processing. In studies of wood (Populus tremuloides or aspen) with 1-ethyl-3-methylimidazolium acetate at room temperature, it was shown that the wood pieces swell by the IL and deswell when treated with water. Applying swelling and deswelling cycles (in the presence of IL and water, respectively), it was possible to incorporate silver and gold nanoparticles in the wood structure (Lucas et al. 2010).

In addition to the dependence on the type of wood and IL, the interaction depends on the water content in both, and hence, one can find apparently quite contradicting results in the published literature (see for instance summaries in Brandt et al. 2010; Gericke et al. 2012). 
Most of the published studies have been carried out with respect to biomass processing requiring dissolution as key step, and only one discusses the kinetics of swelling by following the change in the size of wood pieces (Brandt et al. 2010).

The authors are aware that a 'universal wood sample' does not exist. The study of swelling and mechanical properties of wood is a complex issue and requires, among others, the knowledge of the geographical origin of the tree, the source of the sample within the tree, and moreover the water content, density, pretreatment history, morphology of the particular wood sample prior to the investigation (Lanvermann et al. 2014; Parham and Gray 1984; Pettersen 1984). A statistical analysis has shown that the maximum swelling of wood mainly depends on the density of the wood as well as on the basicity and the molecular volume of the swelling agent (Mantanis et al. 1995b).

The aim of this contribution is not to present as exact as possible swelling data for a particular wood sample, but to report interesting experimental findings which, according to the authors' understanding, might be generally valid. Results are presented with respect to the interaction of spruce and beech wood with water, ILs, and mixtures of both using a recently described experimental procedure to follow the swelling behavior of synthetic polymer gels (Höhne and Tauer 2014). This experimental method is different to the classical experimental techniques following the interaction of wood with solvents which have been developed over the years such as following the dimensional changes by size measurements (Mantanis et al. 1994a, b, 1995a, b) or changes in the wood cells by X-ray tomographic investigations (Derome et al. 2011; Patera et al. 2013) and microscopic studies (Kanbayashi and Miyafuji 2013, 2014, 2015; Miyafuji and Suzuki 2012). One advantage of the method employed in this study is its simplicity which allows monitoring the solvent uptake in situ and in an open environment under varying experimental conditions with respect to temperature, relative humidity, and solvent composition even during a running experiment.

Particularly, the behavior of three ILs was investigated: ethylammonium nitrate (EAN), the first IL ever described (Freemantle 2010), 1-butyl-3-methylimidazolium chloride (BMIMC), and 1-hexyl-3-methylimidazolium chloride (HMIMC). EAN and HMIMC are liquids at room temperature (RT). BMIMC has melting point of about $65{ }^{\circ} \mathrm{C}$ and becomes liquid at RT only after the uptake of water from the air. All three ILs differ with respect to hydrophilicity and viscosity. Moreover, ethylammonium nitrate is a protic IL and, compared with the other two which are aprotic ILs, very similar to water and able to hydrogen bonding (Evans et al. 1982; Allen et al. 1985). It was shown that water is capable of accelerating the uptake of these ILs by wood and that they remain in the wood, whereas water slowly evaporates. In addition, it was proven that wooden connections made by swelling can be tuned to last almost infinitely if ionic liquids are used as swelling agent. In addition, the presence of the ionic liquid in wood reduces the action of water applied subsequently quite drastically. 


\section{Materials and methods}

\section{Materials}

Untreated, room-dried cubes with edge length of 5, 7.5, 11.4, and $20 \mathrm{~mm}$ made of spruce (Picea abies) wood were used for most of the swelling studies. If not otherwise stated, swelling was investigated in radial direction (Fig. 1). Joining studies were carried out with beech wood cylinders (Fagus sylvatica, outer diameter $8 \mathrm{~mm}$ with the long axis in the stem direction perpendicular to the growth rings) and aluminum rings (inner diameter of $8.3 \mathrm{~mm}$ ), see Fig. 1. The wood samples (untreated wood from a hardware store) were not specifically pretreated before the measurements, and their water content was as high as determined by the given temperature and relative humidity in the laboratory. The density at ambient conditions is for the spruce and beech wood used about 0.44 and $0.72 \mathrm{~g} / \mathrm{cm}^{3}$, respectively. The water content was determined to be for both wood types 5.2-5.3 wt \% before use after storage under ambient laboratory conditions. The ILs ethylammonium nitrate (EAN from Roth), 1-butyl-3-methylimidazolium chloride

(a)

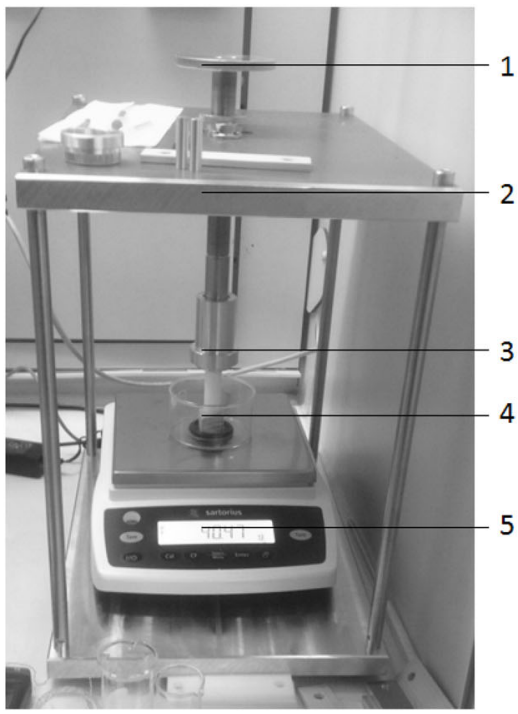

(b)

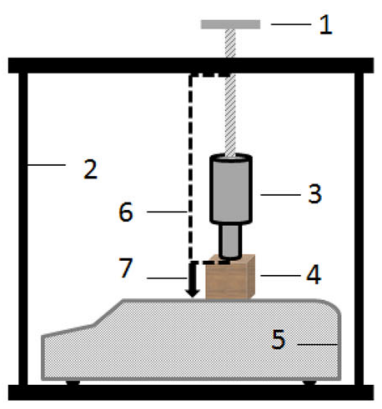

(c)

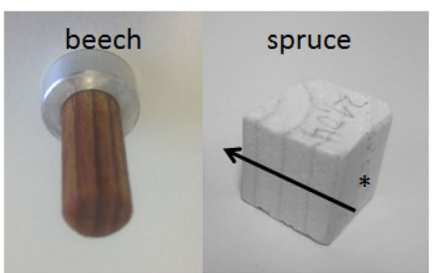

Fig. 1 a Experimental setup (and b schematic drawing) used for measuring the swelling behavior of wood, 1 adjusting knob for the desired starting load, 2 cage to reduce disturbances from vibrations and deformations of the table and to minimize loss of swelling force due to the elastic and springy properties of the materials used for the construction, 3 Teflon stamp in contact with the wood, 4 wood placed in a glass container in the middle of a Viton O-ring, and the swelling agent is placed between the wood and the O-ring, 5 balance connected with a computer, 6 dashed clip marks the fixed distance which cannot change during the experiment, 7 arrow shows the direction of the swelling pressure which causes the balance readout (apparent mass) $\mathbf{c}$ beech cylinder connected to the aluminum ring (left-hand side) and spruce cube with edge length of $20 \mathrm{~mm}$ (right-hand side) used for the corresponding investigations; the arrow crossing the growth rings perpendicularly indicates the direction toward the balance, the arrowhead in (c) and (b) point in the same direction, and the asterisk marks the side where the Teflon stamp was placed 
(BMIMC from Sigma-Aldrich), and 1-hexyl-3-methylimidazolium chloride (HMIMC from Fluka) were used as received. In the liquid state, these ILs have at room temperature a viscosity higher than that of water. Water was taken from a SG purification system (Hamburg) with a conductivity of $0.055 \mu \mathrm{S} \mathrm{cm}^{-1}$.

\section{Measurements}

The force increase connected with the swelling of wood was measured in a device as shown in Fig. 1. The readout of the balances (Entris 6202-1S or Practum 5101-1S both from Sartorius, Germany) was transferred and continuously recorded with the MultiControl software on a computer. Details of the measurements applied to polymeric gels have been recently communicated (Höhne and Tauer 2014).

Two different constructions were used for the fixation of the wood sample on the weighing pan. Setup A (cf. Fig. SI-1a) is a kind of cage construction aimed to minimize losses of the swelling force due to the elasticity of the connecting materials. Setup B is a simpler construction where the balance is directly placed on the laboratory bench and the wood is fixed in place by means of retort stand rods and apparatus clamps (cf. Fig. SI-1b). All measurements were repeated several times (at least two times) with different pieces of wood (swelling with ionic liquids) or repeatedly with the same piece of wood after drying (swelling with water).

The drying process alone (evaporation of water) was recorded gravimetrically with a Mettler Toledo AG204 balance, and the BalanceLink software was used for data transfer to a computer.

\section{Data evaluation}

The weighing method to study swelling of gels uses the relation between the swelling pressure and the weight force Eq. (1). For a discussion of the relation of this method to study swelling of polymeric gels to other methods, the reader is referred to a former publication (Höhne and Tauer 2014). For the data evaluation, one should always consider that the chemical composition of wood fluctuates not only from species to species but for a given species with the particular conditions of growth (nature of soil and climate) and even along a single tree (Pettersen 1984). In this sense, each wood sample is quite a unique object to be examined.

The balance readout is directly related to the swelling pressure (Eq. 1) assuming isochoric conditions. However, this is not strictly fulfilled during the experiment because the wood is not confined in a semipermeable container preventing dimensional changes. Nevertheless, the tightness of the wood produces a pressure, $\Delta \mathrm{P}_{\mathrm{s}}$, which in analogy to the osmotic pressure contributes to the equilibration of the chemical potential of the swelling agent outside and inside the wood, $\mu_{1 \mathrm{o}}$ and $\mu_{1 \mathrm{sg}}$, respectively. Here $v_{\mathrm{m}, \mathrm{sa}}$ is the molar volume of the swelling agent and g denotes the gravitational constant. 


$$
\Delta P_{s}=\frac{1}{v_{m, s a}}\left(\mu_{10}-\mu_{1 s g}\right)=\frac{1}{v_{m, s a}} \Delta \mu_{1}=\frac{\Delta w_{s g}}{a_{g}}=\frac{\Delta m_{s g} \cdot g}{a_{g}}
$$

The mass change related to the swelling pressures, $\Delta \mathrm{m}_{\mathrm{sg}}$, is evaluated as an apparent mass $\left(m_{\text {app }}\right)$ which corresponds to the force generated by the portion of the swelling pressure that is redirected toward the balance. There are always losses of some kinds in this force due the elasticity and springy properties of the construction, the materials involved, and the wood sample itself. So, the application of Eq. (1) allows the calculation of only a portion of the swelling pressure. Indeed, for a spruce cube with an edge length of $20 \mathrm{~mm}$, an apparent mass of about $2 \mathrm{~kg}$ corresponds to $\Delta \mathrm{P}_{\mathrm{s}}$ of about $50 \mathrm{kPa}$ which is expectedly-due to the experimental conditions of noncomplete swelling restrain and real wood samples-far below the theoretically possible maximum swelling pressure of wood (wood assumed as void-free gel; Stamm 1964). For a beech wood cube of the same size (data not shown), the observed swelling pressure in the maximum swelling direction was much higher than the weighing range of the balance $(>6 \mathrm{~kg})$. Throughout this contribution, the primary experimental data will be presented as $m_{\text {app }}$ - time plots.

The chamber where the wood and the swelling agent are placed is open, and hence, normal liquids can evaporate over longer periods of time. This is not the case for ionic liquids because their vapor pressure is at ambient conditions extremely low, measurable for protic ILs at higher temperatures (Greaves and Drummond 2008; Emel'yanenko et al. 2014). One might consider the openness of the measurement chamber as shortcoming, but the authors consider the advantages (possibilities to easily modify the swelling conditions and to implement additional sensors) clearly outweigh the odds.

It should be noted that in contrast to polymer gels where the samples must be confined in a container with walls permeable only for the swelling agent (Höhne and Tauer 2014), the wood pieces were used without confinement. Due to the firmness of the wood, isochoric conditions are preserved to a great deal; at least the small changes that happen do not limit the meaningful conclusions of the measurements.

\section{Results and discussion}

\section{Swelling with water}

Figure 2 illustrates the usefulness of the weighing method by means of the swelling of spruce cubes with variable size. Contrary to synthetic polymeric gels, the size of the wood is a critical issue due to the inhomogeneity of the wood with respect to morphology and chemical composition. Figure $2 \mathrm{a}$ shows the time development of the balance readout for spruce cubes of different size until a constant value is reached. The change in the apparent mass with time appears quite smooth except during the very initial period (Fig. 2b for the first $200 \mathrm{~s}$ ) where the order apparently does not correlate with the cube size but is dominated by the accessibility of the water to the hydrophilic components. Only after about $1 \mathrm{~h}$, the balance readout scales with the size of the cubes. Both the constant apparent mass and the time until 

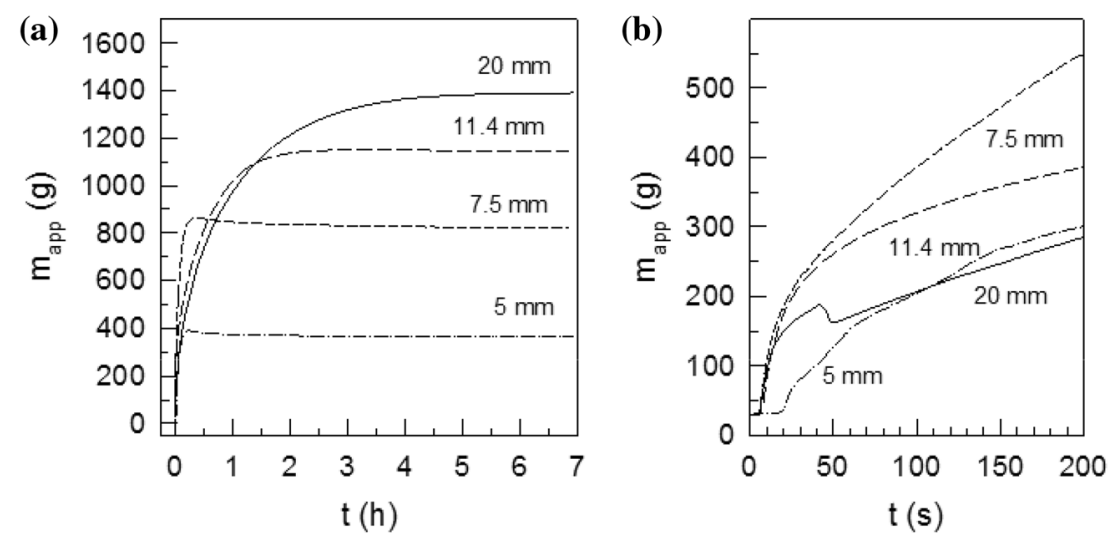

Fig. 2 Temporal change in the balance readout (setup A), $m_{a p p}$, during the swelling of spruce wood cubes with water: a range until a constant value is reached and b initial $200 \mathrm{~s}$; the numbers in the graphs denote the edge length of the corresponding cube; the curves were obtained by adding an eightfold excess of water to the three smaller cubes and $8 \mathrm{ml}$ of water to the largest cube

this state is reached depend on the cube size. Expectedly, the larger the cube, the greater the constant balance readout reached in this steady state. The duration of the steady state depends on the amount of water and is controlled by the evaporation rate.

Besides the amount of water, the setup for fixing the wood on the balance has a strong influence on the balance readout (cf. Fig. SI-2). Expectedly, the readout is in any case larger for a setup which redirects a larger portion of the swelling toward the balance (setup A). This is caused by the construction principle which reduces the losses of the swelling force due to the elastic and springy properties of the metal rods and connections necessary to redirect the force toward the weighing pan much more for setup A than for setup B (cf. Fig. SI-1). Apparently, the observed difference between both setups depends on the cube size and becomes increasingly important the larger the cubes, whereas the amount of water seems to be less important (cf. Fig. SI-2). However, these are trends, but for drawing statistically significant conclusions much more experiments are necessary.

Figure 3 shows correlations derived from averaged data (averaging the data as illustrated in Fig. SI-2) between the swelling behavior and the size of the spruce cubes. The data clearly show that both the measured constant apparent mass and the time until this mass is reached depend on the size of the wood cubes.

It seems intuitively reasonable that both the balance readout (the apparent mass) and the time to reach a constant apparent mass increase with the cube size. According to Eq. (1), one might expect that the apparent mass which is related to the swelling pressure scales with the area of the side surface of the cube. The time until the apparent mass is constant should correspondingly scale with the volume of the cube. Indeed, the experimental data generally proof an increase in both values with the side area and volume of the cube. However, due to the already abovediscussed imperfections of both experimental setups the experimental data deviate 

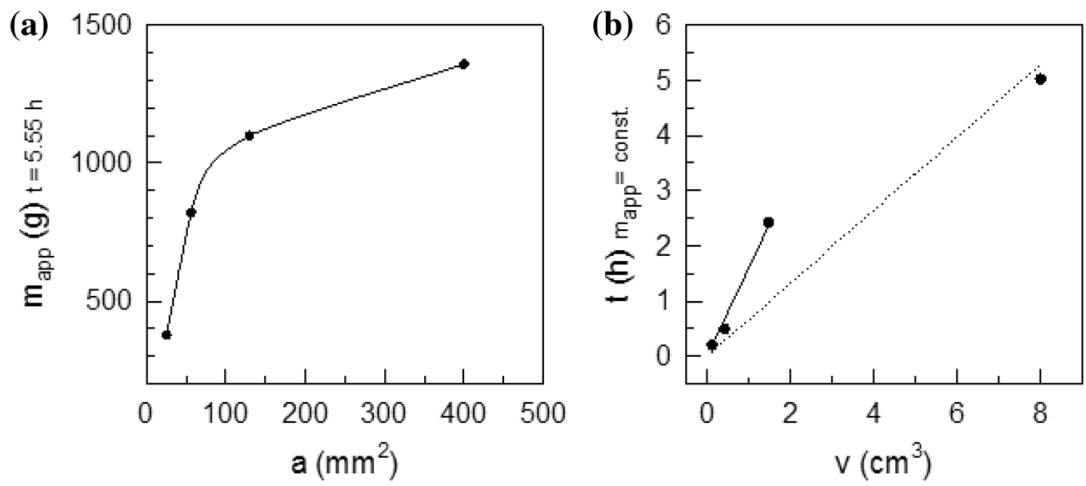

Fig. 3 Correlations derived from the averaged swelling data (depicted in Fig. SI-2); a maximum apparent mass taken $5.55 \mathrm{~h}$ after adding water in dependence on the area of the side of the spruce cube and $\mathbf{b}$ the time until the balance readout reaches a constant value after the water addition in dependence on the volume of the spruce cube, the lines are regression forced through the origin for all data (dotted line) and the data for the three smallest cubes (solid line)

the stronger the larger the cubes, which means that larger portions of the swelling force are lost in the lever rod system of the setup. The data of Fig. $3 b$ show two linear regression lines each forced through the origin. The dotted line, considering all four data points, proves the poor correlation caused by the strong deviation of the data for the largest cube. For the largest cube, the size of the stamp fixing the tube on the balance is smaller than the cube area which is not optimal because an additional portion of the swelling pressure gets lost due to curving of the uncovered upper cube area. This is probably the main reason for the deviation of the data for the largest cube.

Despite the fact that the individual data are prone to quite a significant scatter due to the properties of the wood (anisotropic multi-component composite material) in combination with the imperfections of the measurements, a meaningful data evaluation is possible. The use of bare wood and the renunciation of the embedding in a confinement with stiff but semipermeable walls contribute to the reduction in the measured effect (compared to the theoretical predicted swelling pressure). In addition, under these non-isochoric conditions the dimensions of the cube change perpendicular to the measurement direction (Fig. 1). The specific construction of the measurement system easily allows the change in the composition of the swelling agent such as the addition or removal of components. The development of the forces connected with uptake and release of the swelling agent can be studied as well as the influence of ambient humidity and temperature as demonstrated in the subsequent paragraph.

\section{Swelling with ionic liquid and water}

The swelling behavior of wood with ILs is very different compared with that of water. In contrast to water, for all three ILs studied, the apparent mass decreases after bringing the IL in contact with the wood (cf. Fig. SI-3). This experimental 
observation is similar to the results described in Lucas et al. (2010). The initial decrease lasts a few $1000 \mathrm{~s}$ before the balance readout almost levels off. Initially decreasing apparent mass was observed in every single experiment and might be explained by the hydrophilic nature of the ILs causing deswelling of wood by dehydration.

In order to emphasize the quite different swelling behavior of spruce with water and ILs, Fig. 4 compares directly in one graph the balance readout for water and HMIMC over a period of several days. Compared with HMIMC, water causes a much stronger swelling force illustrated by $m_{\text {app }}$ which is in this particular case within the first day and by about a factor of 10 higher.

The addition of water, at a certain time after the interaction of wood with ILs, drastically increases the apparent mass caused by the swelling power of water (Fig. 4; SI-3). The maximum balance readout is dominated by water and almost independent of the IL. The visual observation of the wood during the different stages of this combined swelling procedure allows the conclusion that the added water also acts as kind of transportation aid for the IL into the wood (cf. Fig. SI-4), because at the end of the experiment (when the water is evaporated) free ionic liquid is not visible any more.

This conclusion is supported by the results presented in Fig. 5 comparing the temporal development of $m_{\text {app }}$ and the weight loss after the addition of water $(m)$ which was done in this experiment almost 4 days after establishing contact between HMIMC and wood. In this graph, in order to ease the comparison, the time axis was set to zero for both plots after the addition of water. The results of both independent measurements prove that swelling of spruce wood with water and evaporation of the water follow different kinetics. Note, for both measurements the experimental situation was comparable: The cube of spruce wood (edge length of

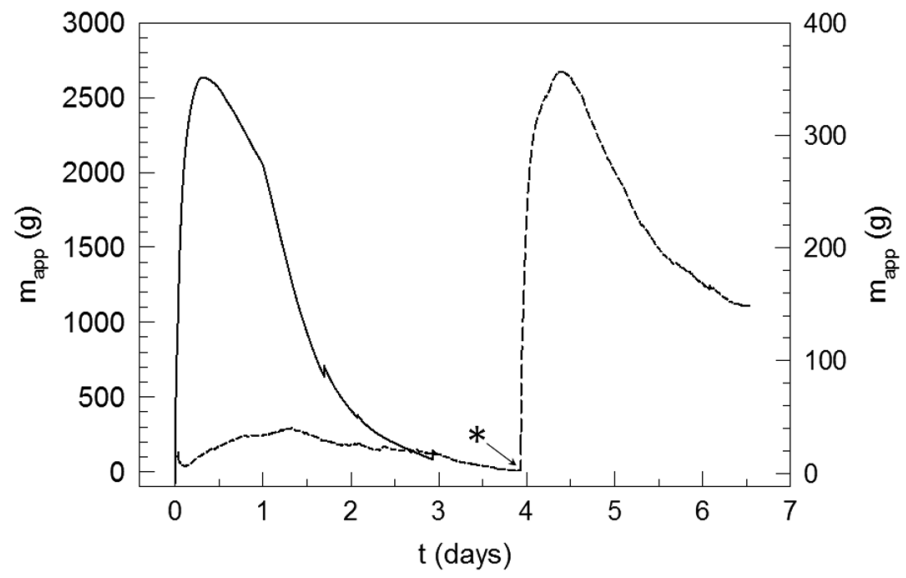

Fig. 4 Apparent mass-time curves for swelling of spruce wood cubes (edge length of $20 \mathrm{~mm}$ ) with HMIMC (dashed line, right y axis) and water (solid line, left y axis); HMIMC or water was added at time zero, and the asterisk marks the addition of water almost 4 days after HMIMC addition; the measurements were carried out with setup A 


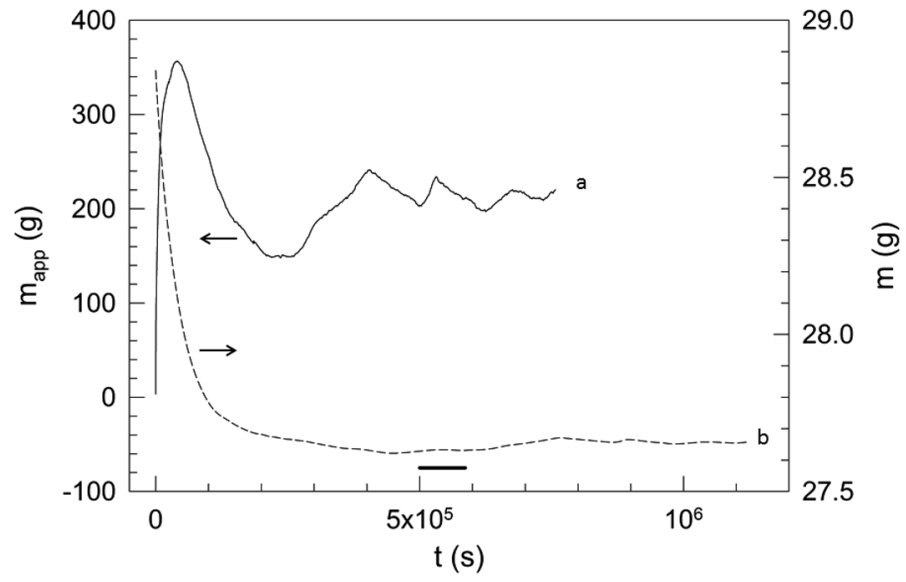

Fig. 5 Following the swelling of a cube of spruce wood (edge length $20 \mathrm{~mm}$ ) with water $(1.1 \mathrm{~g})$ added 4 days after the addition of HMIMC ( $m_{\text {app}}$, solid line $a$, left $y$ axis) and the weight loss ( $m$, dashed line $b$, right $y$ axis); the short solid line parallel to the $x$ axis represents $24 \mathrm{~h}$

$20 \mathrm{~mm}$ ) was placed in a glass container, and the same amount of IL (HMIMC) and water (1.1 g about 4 days later) was added. The whole protocol of the experiment for the observation of the evaporation of water is given in Fig. SI-5.

The water evaporation follows the expected behavior-a smooth decrease-over the first $10^{5} \mathrm{~s}$ (about $28 \mathrm{~h}$ ) which is the time it takes until all the added water has evaporated (curve b). Interestingly, the swelling pressure (or the apparent mass) reaches its maximum when more than $50 \%$ of the water already has been gone. The swelling of the wood relaxes much more slowly than the water evaporation happens ( $m_{\text {app }}$ reaches the minimum value only about $28 \mathrm{~h}$ after the added water has left the cube). The following changes in both curves are due to the changes in the ambient conditions with respect to humidity and temperature which has been proven by simultaneously recording $m_{\text {app }}$, humidity, and temperature (cf. also Fig. SI-6). The balance readout reacts on both, changes in humidity and temperature, however much more pronounced on the former. This result underlines the sensitivity of the weighing method to study the swelling behavior of wood.

Probably the most interesting result revealed by the data put together in Figs. 4 and 5 is the persisting swelling of wood with ILs after the water has completely evaporated. After the evaporation of water, the swelling pressure $\left(m_{\mathrm{app}}\right)$ stays much higher than the starting value. This finding is of some practical relevance because it allows the construction of durable joints-containing wooden elements. Moreover, this behavior is qualitatively different from that of water as swelling agent because ILs are practically not evaporable and even direct contact of the joints with water is not harmful since the connection is tightened.

Another interesting conclusion regarding the swelling behavior of wood preswollen with IL can be drawn from the curves compared in Fig. 4. Both curves show the swelling of wood with water. However, the natural untreated wood swells much stronger, under otherwise identical conditions, by almost one order of 
magnitude. This observation is highly reproducible (cf. Fig. SI-6), but its origin is not completely clear at the moment. Also the following observation might be causally connected with that. The evaporation of water from wood pretreated with IL is faster than from pure wood (cf. Fig. SI-7). Here it should be noted that compared to the evaporation of water from the neat glass container, the presence of pure wood delays the evaporation and changes the shape of the mass-time curve. Interestingly, the presence of IL in the wood accelerates the evaporation; however, it is still slower than from the empty glass container.

With respect to a consistent explanation for these observations, it seems reasonable that the water inside the pure wood (not containing ILs) is withheld in the hydrophilic regions, thus slowing down evaporation. Possibly, in wood pretreated with IL, the internal morphology has changed in a way that penetration of water into the wood is hindered and/or certain paths for the water to the hydrophilic regions are blocked. Consequently, the rate of evaporation is increased and the swelling of wood is reduced. This conclusion seems reasonable since changes in the morphology of the anisotropic structure of wood tissues during the interaction with ILs have been described (Miyafuji and Suzuki 2012; Kanbayashi and Miyafuji 2013, 2014).

The different behavior of joints-containing wood either pre-swollen with water or IL is illustrated in Fig. 6 (cf. also movie SI-1). Even storage in a vacuum oven (pressure of $1 \mathrm{mbar}$ at $40{ }^{\circ} \mathrm{C}$ ) for several weeks obviously does not change the swelling of the wood with IL. Figure 6 exemplifies this by means of beech wood cylinders which were connected to aluminum rings by swelling, one with water and the other with IL (here EAN).

The images of Fig. 6 illustrate nicely that water and EAN behave on the one hand qualitatively similar and on the other hand quantitatively completely different. Before placing the assemblies in the vacuum oven, the wooden cylinders were
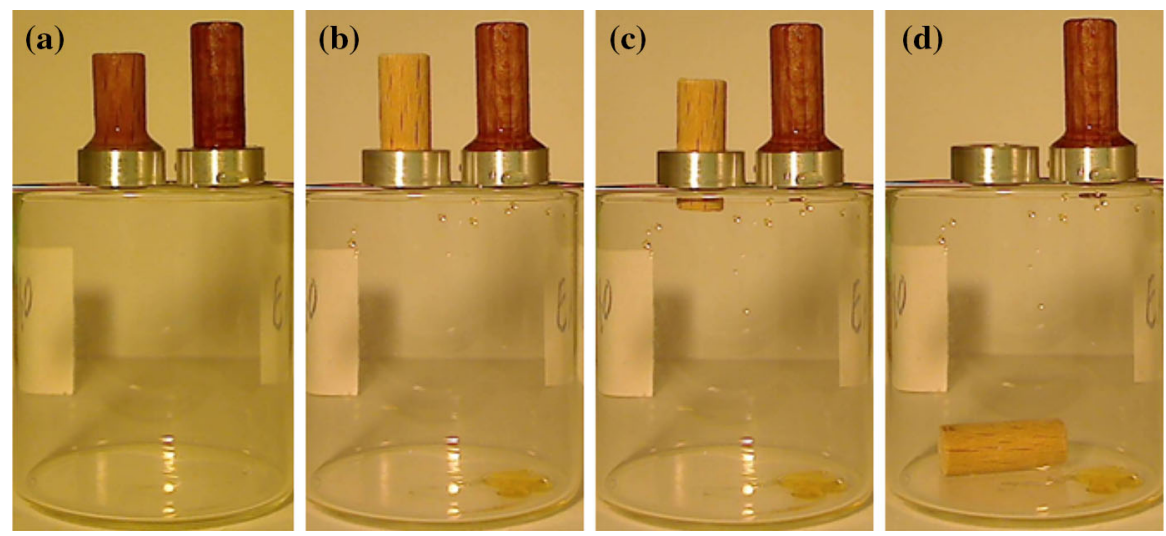

Fig. 6 Snapshots illustrating the behavior of a wooden cylinder (beech) embedded in an aluminum ring by swelling of the wood with water (assembly to the left) and EAN (assembly to the right) in a vacuum oven (operating with a membrane pump) at pressure and temperature of 1 mbar and $40{ }^{\circ} \mathrm{C}$, respectively; the assemblies are mounted on top of a glass beaker; a image taken after evacuation, $\mathbf{b}$ image taken $1 \mathrm{~h}$ later, c image taken $1 \mathrm{~h}$ and 25 min later, $\mathbf{d}$ image taken $1 \mathrm{~h}$ and $27 \mathrm{~min}$ later 
swollen under ambient conditions to equilibrium by dipping them stuck into the aluminum ring in the corresponding liquids. Expectedly by reducing the pressure, the excess swelling agent is released and appears, for both liquids, as free phase adhering to the wood-aluminum-connecting edge. This happens for both systems, however much faster for water than EAN (compare images a and b). Simultaneously, but visible here only for water, evaporation takes place leading to the disappearance of the free liquid phase followed by drying and shrinking of the wood and eventually the beech cylinder slips through the aluminum ring. Although the vapor pressure of EAN is non-negligible, it is, however, much lower and its viscosity is about a factor of almost 100 higher than that of water (Belieres and Angell 2007). Hence, the images of Fig. 6 do not show any signs of a decreasing amount of EAN at the wood-aluminum juncture. The droplets visible at the wall of the glass beaker are splashes stemming either from disrupting air bubbles, covered by a thin layer of EAN, which are leaving the wood during evaporation, or fallen EAN-drops. The connection between the wood swollen with water and aluminum breaks after $1 \mathrm{~h}$ and $27 \mathrm{~min}$, whereas the connection between wood swollen with EAN and aluminum lasts apparently 'forever,' even under vacuum.

\section{Conclusion}

The weighing method as described recently (Höhne and Tauer 2014) is an easy to use and versatile tool to study the swelling behavior of wood. The measuring system is open, and hence, during the measurements the composition of the swelling agent can be changed without interrupting the data recording. This feature allows transient measurements, i.e., following uptake and release of volatile swelling agents. Studying the swelling of wood samples with a mixture of ionic liquids and water revealed a particular behavior. On the one hand water swells wood much faster and stronger than the ionic liquid alone, and on the other hand it facilitates the uptake of the ionic liquid. Transient studies have shown that water evaporates alone, but the ionic liquid remains in the wood causing a sustained swelling. The durable swelling with the ionic liquid is useful for the construction of permanent joints in wood-containing assemblies. Another benefit for the application of wooden construction can be expected with respect to antifouling and antibacterial protection because it is known that ionic liquids are effective in this sense (Ye et al. 2012; Zhang et al. 2014).

Acknowledgments Open access funding provided by Max Planck Society (or associated institution if applicable). We thank J. von Szada, M. Bott, G. Haseloff from the mechanical workshop, and K. Bienert from the electronic workshop of the MPI for careful production of the experimental setup for measuring the swelling pressure (apparent mass). Experimental assistance of Mr. Chunxiang Wei is gratefully acknowledged. P.H. acknowledges a fellowship and financial support within the DFG priority program SPP1420.

Open Access This article is distributed under the terms of the Creative Commons Attribution 4.0 International License (http://creativecommons.org/licenses/by/4.0/), which permits unrestricted use, distribution, and reproduction in any medium, provided you give appropriate credit to the original author(s) and the source, provide a link to the Creative Commons license, and indicate if changes were made. 


\section{References}

Allen M, Evans DF, Lumry R (1985) Thermodynamic properties of the ethylammonium nitrate + watersystem - partial molar volumes, heat-capacities, and expansivities. J Solut Chem 14(8):549-560

Belieres JP, Angell CA (2007) Protic ionic liquids: preparation, characterization, and proton free energy level representation. J Phys Chem B 111(18):4926-4937

Brandt A, Hallett JP, Leak DJ, Murphy RJ, Welton T (2010) The effect of the ionic liquid anion in the pretreatment of pine wood chips. Green Chem 12(4):672-679

Chowdhury KA (1970) Wood and its use during pre- and proto-historic time. J Hist Sci 5(1):141-143

Derome D, Griffa M, Koebel M, Carmeliet J (2011) Hysteretic swelling of wood at cellular scale probed by phase-contrast X-ray tomography. J Struct Biol 173(1):180-190

Emel'yanenko VN, Boeck G, Verevkin SP, Ludwig R (2014) Volatile times for the very first ionic liquid: understanding the vapor pressures and enthalpies of vaporization of ethylammonium nitrate. ChemEur J 20(37):11640-11645

Evans P (2003) Emerging technologies in wood protection. Forest Prod J 53(1):14-22

Evans DF, Yamauchi A, Roman R, Casassa EZ (1982) Micelle formation in ethylammonium nitrate, a low-melting fused salt. J Colloid Interface Sci 88(1):89-96

Freeman MH, Shupe TF, Vlosky RP, Barnes HM (2003) Past, present, and future of the wood preservation industry. Forest Prod J 53(10):8-15

Freemantle M (2010) An introduction to ionic liquids 2. RSC, Cambridge

Gericke M, Fardim P, Heinze T (2012) Ionic liquids-promising but challenging solvents for homogeneous derivatization of cellulose. Molecules 17(6):7458-7502

Greaves TL, Drummond CJ (2008) Protic ionic liquids: properties and applications. Chem Rev 108(1):206-237

Han SQ, Li JL, Zhu SD, Chen R, Wu YX, Zhang XY, Yu ZN (2009) Potential applications of ionic liquids in wood related industries. Bioresources 4(2):825-834

Höhne P, Tauer K (2014) How much weighs the swelling pressure. Colloid Polym Sci 292(11):2983-2992

Kanbayashi T, Miyafuji H (2013) Morphological changes of Japanese beech treated with the ionic liquid, 1-ethyl-3-methylimidazolium chloride. J Wood Sci 59(5):410-418

Kanbayashi T, Miyafuji H (2014) Comparative study of morphological changes in hardwoods treated with the ionic liquid, 1-ethyl-3-methylimidazolium chloride. J Wood Sci 60(2):152-159

Kanbayashi T, Miyafuji H (2015) Topochemical and morphological characterization of wood cell wall treated with the ionic liquid, 1-ethylpyridinium bromide. Planta. doi:10.1007/s00425-014-2235-7

Lanvermann C, Hass P, Wittel FK, Niemz P (2014) Mechanical properties of norway spruce: intra-ring variation and generic behavior of earlywood and latewood until failure. Bioresources 9(1):105-119

Lucas M, Macdonald BA, Wagner GL, Joyce SA, Rector KD (2010) Ionic liquid pretreatment of poplar wood at room temperature: swelling and incorporation of nanoparticles. Acs Appl Mater Interfaces 2(8):2198-2205

Mantanis GI, Young RA, Rowell RM (1994a) Swelling of wood. 1. Swelling in water. Wood Sci Technol 28(2):119-134

Mantanis GI, Young RA, Rowell RM (1994b) Swelling of wood. 2. Swelling in organic liquids. Holzforschung 48(6):480-490

Mantanis GI, Young RA, Rowell RM (1995a) Swelling of wood. 3. Effect of temperature and extractives on rate and maximum swelling. Holzforschung 49(3):239-248

Mantanis GI, Young RA, Rowell RM (1995b) Swelling of wood. 4. A statistical-model for prediction of maximum swelling of wood in organic liquids. Wood Fiber Sci 27(1):22-24

Miyafuji H, Suzuki N (2012) Morphological changes in sugi (Cryptomeria japonica) wood after treatment with the ionic liquid, 1-ethyl-3-methylimidazolium chloride. J Wood Sci 58(3):222-230

Narasimhan B, Peppas NA (1997) The physics of polymer dissolution: modeling approaches and experimental behavior. Adv Polym Sci 128:157-207

Parham RA, Gray RL (1984) Formation and structure of wood. Adv Chem Ser 207:3-56

Patera A, Derome D, Griffa M, Carmeliet J (2013) Hysteresis in swelling and in sorption of wood tissue. J Struct Biol 182(3):226-234

Pettersen RC (1984) The chemical-composition of wood. Adv Chem Ser 207:57-126

Rowell RM (2005) Moisture properties. In: Rowell RM (ed) Handbook of wood chemistry and wood composites. Taylor \& Francis, Boca Raton, pp 77-98 
Rowell RM, Banks WB (1985) Water repellency and dimensional stability of wood. US Government Printing Office:1985/641-044/20015 edn. US Department of Agriculture

Rowell RM, Pettersen R, Han JS, Rowell JS, Tshabala MA (2005) Cell wall chemistry. In: Rowell RM (ed) Handbook of wood chemistry and wood composites. Taylor \& Francis, Boca Raton, pp 35-74

Schultz TP, Nicholas DD, Preston AF (2007) Perspective-a brief review of the past, present and future of wood preservation. Pest Manag Sci 63(8):784-788

Stamm AJ (1964) Shrinking and swelling of wood. Wood and cellulose science. The Ronald Press Company, New York, pp 215-235

Swatloski RP, Spear SK, Holbrey JD, Rogers RD (2002) Dissolution of cellose with ionic liquids. J Am Chem Soc 124(18):4974-4975

Ye Q, Gao TT, Wan F, Yu B, Pei XW, Zhou F, Xue QJ (2012) Grafting poly(ionic liquid) brushes for anti-bacterial and anti-biofouling applications. J Mater Chem 22(26):13123-13131

Zhang TL, Zhu CY, Ma HM, Li RY, Dong BG, Liu YF, Li SZ (2014) Surface modification of APA-TFC membrane with quaternary ammonium cation and salicylaldehyde to improve performance. J Membr Sci 457:88-94 